\title{
Isolation and Characterization of Chicken NPAS3
}

\author{
Jiheon Shin, Hye Yun Jeong, Kyung Eun Lee and Jaesang Kim* \\ Division of Life and Pharmaceutical Sciences, Ewha Womans University, \\ Seoul 120-750, Korea
}

\begin{abstract}
Here we describe characterization of chicken neuronal Per-Arnt-Sim domain 3 (NPAS3) gene during embryogenesis including examinations of expression pattern and function of the gene. RTPCR assay showed that the primary tissue of expression for this gene is the central nervous system (CNS) while RNA in situ hybridization assay confirmed that NPAS3 was expressed in the ventricular zone of developing neural tube as early as Hamburger-Hamilton $(\mathrm{HH})$ stage 20. Ectopic over-expression of the gene in ovo in the developing chicken neural tube by electroporation had little effect on stem cell population, overall neurogenesis, and motor neuron differentiation. We discuss the implications of our observation.
\end{abstract}

Key words: NPAS3, CNS, neural tube, chicken, in ovo electroporation

\section{INTRODUCTION}

Neuronal PAS domain protein 1 (NPAS1) and NPAS3 belong to the basic helix-loop-helix (bHLH) family of transcription factors (Erbel-Sieler et al., 2004). The presence of PAS (Per-Arnt-Sim) domain, which mediates protein-protein interaction, defines this subfamily within the larger bHLH gene family (Partch and Gardner, 2010). NPAS3 has recently gained much attention due to its possible association with schizophrenia. Specifically, a translocation between chromosomes 9 and 14, resulting in disruption of NPAS3, has been associated with schizophrenia and learning disability (Pickard et al., 2005; Pickard et al., 2006). In addition, NPAS3 knockout mice show behavioral abnormalities, indicating a possible developmental and regulatory role of NPAS3 that

\footnotetext{
*To whom correspondence should be addressed. TEL: 82-2-3277-3414, FAX: 82-2-3277-3760

e-mail: jkim1964@ewha.ac.kr

Received September 20, 2010

Accepted for publication September 29, 2010
}

may be etiologically relevant to various forms of psychosis (Erbel-Sieler et al., 2004). Such hypothesis is further bolstered by that NPAS3 is expressed in inhibitory interneuron (Erbel-Sieler et al., 2004) and that NPAS3 knockout mice show significant deficiency in adult neurogenesis in the hippocampal dentate gyrus (Pieper et al., 2005).

Remarkably, little is known about the function of NPAS3 during embryogenesis. This is despite the fact that NPAS3 is widely expressed in the developing CNS in mouse (Brunskill et al., 1999) and that adult-stage phenotype of the NPAS3 knockout mouse may be affected by early developmental role of NPAS3. Clearly, the lack of visible neural phenotype during development limited extent of interest thus far, and other approaches than gene targeting in mouse may be required for functional analysis of NPAS3. To this end, we sought to use the chicken system in which neural tube development and neurogenesis therein are relatively well characterized and gain-of-function assays are possible (Nakamura and Funahashi, 2001). We present our preliminary results 
from RTPCR, RNA in situ hybridization and in ovo electroporation assays.

\section{MATERIALS AND METHODS}

\section{RTPCR}

Total RNA preparations were obtained from neural tube, heart, liver and limb of a HH stage 29 chicken embryo with TRIzol (Invitrogen). Random hexamerprimed reverse transcription was performed on each RNA preparation with SuperScript ${ }^{\mathrm{TM}}$ First-Strand Synthesis System for RT-PCR (Invitrogen) following the manufacturer's protocol. First-strand cDNAs thus generated served as templates for PCR amplification. For the amplification of NPAS3 CDNA, a pair of primers, 5'-AGTTGTTGCCACTGCCTGC-3' and 5'-CATGTGGCAGTCAATCCTGAC-3', was used for a single 22-cycle round of PCR. For GAPDH, primers were 5'-GAAACCAGCCAAGTATGATGA-3' and 5'-T CACTCCTTGGATGCCATG-3'. The annealing temperature was $58^{\circ} \mathrm{C}$.

\section{RNA in situ hybridization}

RNA in situ hybridization on frozen embryo sections was performed using the methods described by $\mathrm{Ma}$ et al. (1998) with minor modifications. Detailed protocol is available upon request. The probe template for NPAS3 was derived from the RTPCR assay described above. The probe for Sox2 was derived from a full length chicken Sox2 cDNA clone (Bylund et al., 2003). Embryo sections were from the forelimb level.

\section{In ovo electroporation and immunohistochemistry}

In ovo electroporation into chicken embryo was performed using methods described by Nakamura and Funahashi (2001) with minor modifications. Detailed protocol is available upon request. Full length Xenopus NPAS3 cDNA (accession number: BC123929) in PCMV-SPORT6 was purchased from Open Biosystems. A GFP expression plasmid, pEGFP-N1 (Clontech) was co-electroporated for assessment of electroporation. Embryos were harvested 48 hours after electroporation, fixed in $4 \%$ paraformaldehyde and embedded in $30 \%$ sucrose and OCT prior to sectioning. Primary antibodies used and titres were: mouse anti-GFP (Molecular Probes, $1: 400$ ), mouse anti-Tuj1 (Covance, 1:400), mouse anti-isl1/2 (De- velopmental Studies Hybridoma Bank, $1: 20$ ) and rabbit anti-Sox2 (Millipore, $1: 500$ ). Secondary antibodies were goat anti-rabbit-lgG Alexa 594 and goat anti-mouse-IgG Alexa 594 (Molecular Probes). Embryo sections were from the forelimb level.

\section{RESULTS}

We first sought to examine the spatiotemporal expression pattern of chicken NPAS3. Based on the predicted chicken NPAS3 sequence (NCBI Reference Sequence: XM_421232.2), we were able to designed a pair of oligonulcleotide primers that spanned multiple exons for PCR amplification of a partial CDNA fragment. For RTPCR examination, we prepared cDNAs from neural tube, heart, liver, and limbs of Hamburger-Hamilton stage 29 chicken embryo. A strong expression was readily detected from the neural tube while a much weaker expression was seen in limb and heart but no expression was seen in liver (Fig. 1). This is consistent with the neural tissue being the principal tissue of NPAS3 expression during embryogenesis.

For more detailed examination of spatiotemporal expression pattern, we utilized RNA in situ hybridization. Embryos from $\mathrm{HH}$ stages 20 and 25 were examined. Sox2, a transcription factor expressed in the ventricular zone of developing CNS was used for comparison. Clear expression was seen in the ventricular zone of neural tube for both Sox2 (Fig. 2A) and NPAS3 (Fig. 2B) in HH stage 20 embryo. The two genes showed significant overlap in expression. HH stage 25 embryo also showed a similar pattern of overlap (Fig. 2C, D) for both genes although by $\mathrm{HH}$ stage 25 the relative extent of expression significantly dwindled for both genes.

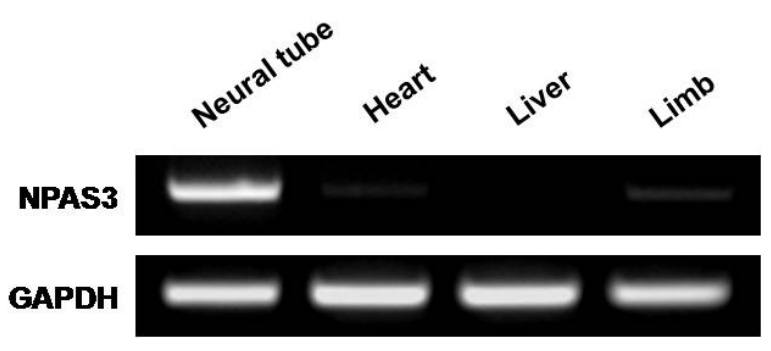

Fig. 1. RTPCR analysis of the NPAS3 expression. Agarose gel electrophoresis of the PCR products is shown. cDNAs derived from indicated tissues were amplified with gene specific primers for NPAS3 and GAPDH. 


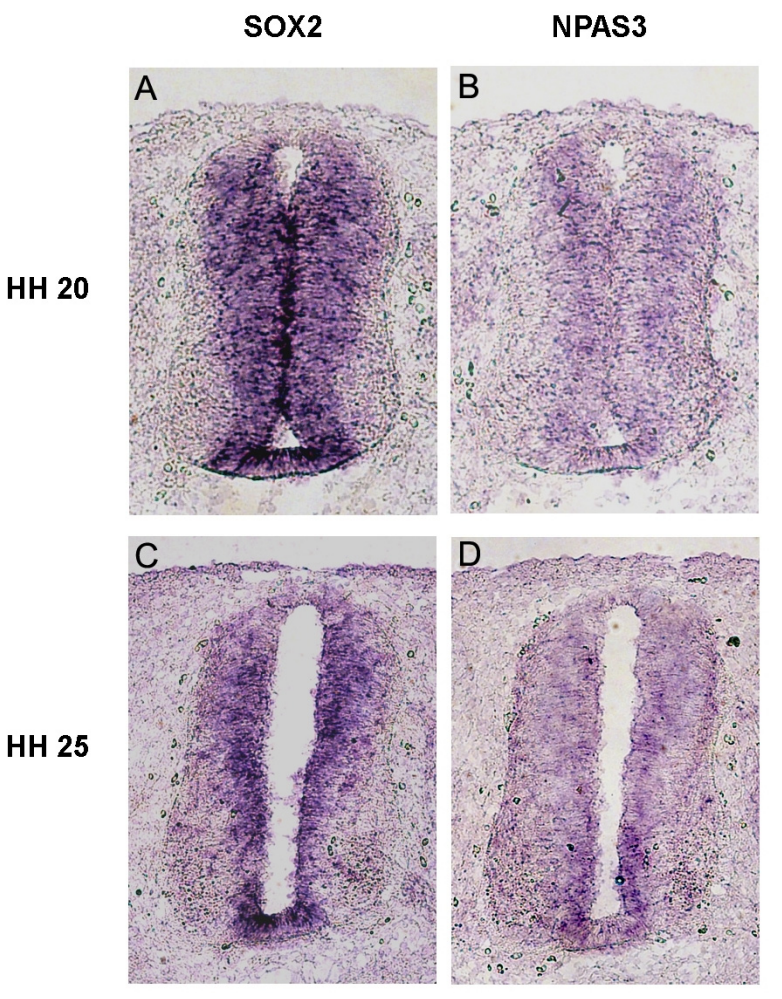

Fig. 2. NPAS3 expression in developing neural tube of $\mathrm{HH}$ stage 20 ( $\mathrm{A}, \mathrm{B})$ and $\mathrm{HH}$ stage 25 (C, D) chicken embryo examined by RNA in situ hybridization assay. The adjacent sections shown are hybridized with antisense RNA probes for Sox2 (A, C) and NPAS3 (B, D). The former was used as a marker of the ventricular zone of developing neural tube.

These results are highly reminiscent of the expression pattern seen in mouse embryos (Brunskill et al., 1999) and indicate that function during neurogenesis is likely conserved.

As the full length cDNA for chicken NPAS3 is not as yet available, we obtained from a commercial source an expression plasmid vector harboring the full length Xenopus laevis NPAS3 cDNA. The plasmid was electroporated into one side of the neural tube along with a plasmid expressing EGFP. The expression of EGFP was readily detected indicating a successful electroporation (Fig. $3 \mathrm{~A}$ ). In this preliminary study, we sought to determine the gross effect of NPAS3 over-expression on neurogenesis by immunostaining for Sox2 (Fig. 3B), a marker for undifferentiated neural stem cells, Tuj1 (Fig. 3C), a pan-neuronal marker, and Isl1 (Fig. 3D), a marker for motor neurons which are the predominant neuronal type produced during early CNS development. Compared to the contra-lateral control side, little if any alterations in expression of the above three

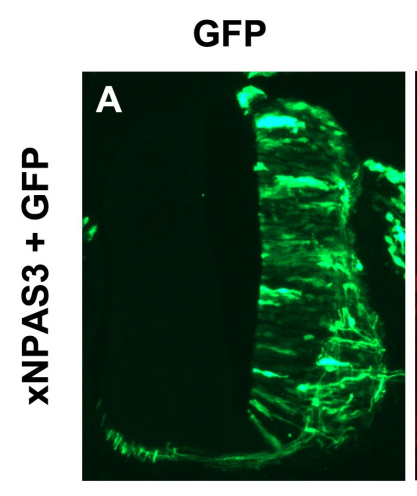

Tuj1

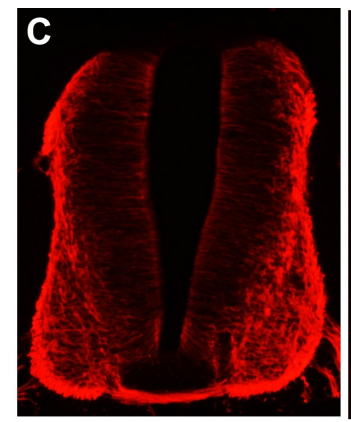

\section{Sox2}

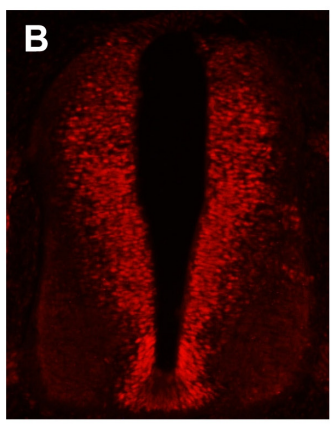

$|s| 1 / 2$

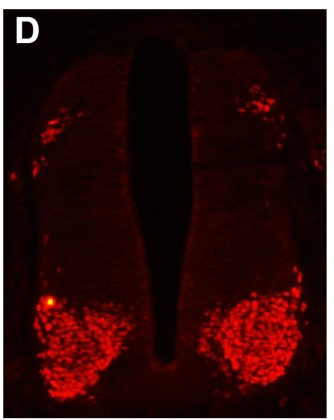

Fig. 3. Expression of neural tube cell markers shows unaltered expression after over-expression of NPAS3. (A) GFP was used as a marker for electroporation. (B) Sox2 positive cells represent neural stem cells. (C, D) Tuj1 is a pan-neuronal marker, and Isl1/2 marks differentiated motor neurons.

markers resulted from NPAS3 over-expression (see below).

\section{DISCUSSION}

All functional analyses of a given gene in a given in vitro or in vivo system have inherent limitations. For example, reporter assay, a representative in vitro assay for analyzing function of transcription factors typically utilizes partial target promoter, heterologous host cell, and over-expression of the transcription factor. Even gene targeting in vivo, typically carried out using mouse, has problems. A frequently encountered one is the lack of phenotypic alterations usually attributed to the functional redundancy of homologues. Another is the problem of early lethality which can prevent spatiotemporal functional analysis. Even more subtle problem is distinguishing between actual function of the targeted gene and the indirect consequences of lacking the gene from the beginning of development. 
In this regard, additional functional assays that can avoid problems from other assays should represent a valuable complement. In ovo electroporation using chicken embryos has long been used for examining gene function in the developing central nervous system, particularly in the neural tube (Jessell, 2000). The accessibility of in vivo tissue for de facto genetic manipulation meant availability of spatiotemporally-specific technique in analyzing gene function in developing neural system. Numerous genes involved in proliferation and differentiation, particularly transcription factors, have been functionally characterized in developing chicken neural tube (Jessell, 2000).

Here, we have shown for the first time that chicken NPAS3 is expressed in the ventricular zone of developing central nervous system. The expression pattern is highly similar to that of mouse which in turn strongly indicates functional conservation. We have also examined the potential role of NPAS3 by ectopically expressing NPAS3 into the developing neural tube. The results indicate that the effect of over-expression on gross neurogenesis at this stage is minimal. One of the strong possibilities is that NPAS3 is already at a saturating level, and the presence of additional NPAS3 has little effect as a consequence. Another possibility is that NPAS3 regulates not overt differentiation of neurons but expression of subset of genes therein. In fact, it has been reported that although NPAS3 is expressed in inhibitory neurons, viability and anatomical distribution of these neurons are not affected in NPAS3 $-1-$ mouse (Erbel-Sieler et al., 2004). Rather gene targeting led to diminished expression of reelin, a protein secreted from inhibitory neurons (Erbel-Sieler et al., 2004). Thus, the results from this preliminary study notwithstanding, a careful gene expression study should be carried out down the road to determine the function of NPAS3. All together, our results establish basic conditions which justify using chicken for future functional analysis of
NPAS3 during embryonic development.

\section{ACKNOWLEDGEMENT}

This study was supported by grants from Brain Research Center of the 21st Century Frontier Research Program (2010K000803) to JK.

\section{REFERENCES}

Brunskill EW, Witte DP, Shreiner AB and Potter SS (1999) Characterization of Npas3, a novel basic helix-loop-helix PAS gene expressed in the developing mouse nervous system. Mech Dev 88:237-241.

Bylund M, Andersson E, Novitch BG and Muhr J (2003) Vertebrate neurogenesis is counteracted by Sox1-3 activity. Nat Neurosc 6:1162-1168.

Erbel-Sieler C, Dudley C, Zhou Y, Wu X, Estill SJ, Han T, Diaz-Arrastia R, Brunskill EW, Potter SS and McKnight SL (2004) Behavioral and regulatory abnormalities in mice deficient in the NPAS1 and NPAS3 transcription factors. Proc Natl Acad Sci USA 101:13648-13653.

Jessell TM (2000) Neuronal specification in the spinal cord: inductive signals and transcriptional codes. Nat Rev Genet 1:20-29.

Ma Q, Chen Z, del Barco Barrantes I, de la Pompa JL and Anderson DJ (1998) neurogenin1 is essential for the determination of neuronal precursors for proximal cranial sensory ganglia. Neuron 20:469-482.

Nakamura $\mathrm{H}$ and Funahashi $\mathrm{J}$ (2001) Introduction of DNA into chick embryos by in ovo electroporation. Methods 24: 43-48.

Partch CL and Gardner KH (2010) Coactivator recruitment: a new role for PAS domains in transcriptional regulation by the bHLH-PAS family. J Cell Physiol 223:553-557.

Pickard BS, Malloy MP, Porteous DJ, Blackwood DH and Muir WJ (2005) Disruption of a brain transcription factor, NPAS3, is associated with schizophrenia and learning disability. Am J Med Genet B Neuropsychiatr Genet 136B: 26-32.

Pickard BS, Pieper AA, Porteous DJ, Blackwood DH and Muir WJ (2006) The NPAS3 gene-emerging evidence for a role in psychiatric illness. Ann Med 38:439-448.

Pieper AA, Wu X, Han TW, Estill SJ, Dang Q, Wu LC, Reece-Fincanon S, Dudley CA, Richardson JA, Brat DJ and McKnight SL (2005) The neuronal PAS domain protein 3 transcription factor controls FGF-mediated adult hippocampal neurogenesis in mice. Proc Natl Acad Sci USA 102:14052-14057. 